Pacific Journal of Mathematics

POINTLIKE SUBSETS OF A MANIFOLD 


\section{POINTLIKE SUBSETS OF A MANIFOLD}

\section{O. Christenson and R. P. Osborne}

Morton Brown introduced the concept of a cellular subset of $S^{n}$. As a consequence of the generalized Schoenflies Theorem it is easy to show that a subset of $S^{n}$ is pointlike if and only if it is cellular. In this paper the obvious generalization of the definitions of pointlike and cellular sets are made and thier relationship in a manifold is considered. It is easy to show that a cellular subset of a manifold is pointlike. While it is not true that a pointlike subset of a manifold is cellular, it is shown that a pointlike subset of a compact $n$-manifold lies in a contractible $n$-manifold with $(n-1)$-sphere boundary. As a consequence of this it is shown that $K$ is a pointlike subset of a compact $n$-manifold $(n \neq 4)$ if and only if $K$ is cellular. The case $n=4$ is still unsolved.

Definitions. An n-manifold is a connected separable locally Euclidean metric space. A connected separable metric space in which every point has a neighborhood whose closure is an $n$-cell is an $n$-manifold with boundary. Note that a manifold is a manifold with boundary boundary but not conversely. A compact connected subset $K$ of an $n$-manifold $M$ is pointlike if $M \sim K$ is homeomorphic with $M \sim\{p\}$ where $p \in M$. A subset $K$ of an $n$-manifold $M$ is cellular if there is a sequence of $n$-cells $C_{1}, C_{2}, \cdots$ such that $C_{i+1} \subset$ Int $C_{i}$ and $K=\cap C_{i}$. An $(n-1)$-sphere $S^{n-1}$ that separates an $n$-manifold $M$ into components $A$ and $B$ is collared on the side containing $A$ if there is an embedding $h: S^{n-1} X[0,1] \rightarrow \bar{A}$ such that $h(x, 0)=x$. An $(n-1)$-sphere $S^{n-1}$ in an $n$-manifold $M$ is bicollared if there is an embedding $h: S^{n-1} X[0,1] \rightarrow M$ such that $h(x, 1 / 2)=x$. A pseudo-sphere is a compact manifold that is a homotopy sphere. A compact contractible $n$-manifold with boundary is called a pseudo-cell. The Poincare Conjecture-known to be true for $n \neq 3,4$ [7]-says that a pseudo-sphere is a sphere.

Preliminary Theorems. The following theorem follows from the corresponding theorem for $E^{n}$ which is proved by the same methods as used in [4].

THEOREM 1. A cellular subset of a manifold is pointlike.

One might think that a pointlike subset of a manifold is cellular. That this is not the case is shown by the following example.

Example 1. Let $M$ be $E^{3}$ minus the integers on the positive $x$-axis, and minus 1 -spheres of radius $1 / 4$ centered at the negative 
integers on the $x$-axis. The 1 -sphere of radius $1 / 4$ and center at 0 is pointlike but not cellular. A similar construction using linked 1-spheres gives an example of a pointlike subset of a manifold containing a loop that is homotopically nontrivial in the manifold. A cellular subset of a manifold is not necessarily contractible, for example the crumpled cube bounded by the Alexander Horned sphere is not simply connected even though it is cellular.

Lemma 2. Let $K$ be a pointlike subset of a compact manifold $M$ with boundary. Let $h^{\prime}: M \sim K \rightarrow M \sim\{p\}$ be a homeomorphism. Then $h^{\prime}$ can be extended to a continuous map $h: M \rightarrow M$ such that $h^{-1}(p)=K$.

Proof. Define $h$ by

$$
h(x)= \begin{cases}h^{\prime}(x) & \text { for } x \in M \sim K, \\ p & \text { for } x \in K .\end{cases}
$$

Let $U$ be an open neighborhood of $p$. Then $\sim U$ is compact; hence, $h^{-1}(\sim U)$ is compact so $M \sim h^{-1}(\sim U)$ is open. Clearly this set contains $K$. Thus $h$ is continuous.

LeMma 3. If $K$ is a pointlike subset of a compact n-manifold $M$ with boundary and $K$ lies in an open $n$-cell, then $K$ is cellular.

Proof. We shall show that if $U$ is a neighborhood of $K$ then there is an $n$-cell $C$ such that $K \subset$ Int $C \subset U$. Using this a simple inductive argument completes the proof. Let $h: M \rightarrow M$ be the continuous map given by the previous lemma. Then $h(U)$ is a neighborhood of $p$. Let $C^{\prime}$ be an $n$-cell with bicollared boundary in $h(U)$ containing $p$ in its interior. Then $h^{-1}\left(C^{\prime}\right)=C$ is a cell by the Generalized Schoenflies theorem.

By obvious modifications of the proof in [8], the Jordan-Brouwer Theorem can be shown to hold in a pseudo- $n$-sphere. Let $K$ be the closure of one of the complementary domains of $S^{n-1}$. If an $n$-cell is sewn to $K$ the result is another pseudo-sphere. Applications of the Van Kampen Theorem, the Mayer-Vietoris Sequence and the Hurewicz Isomorphism show that $K$ is $(n-2)$-connected. Theorem 6.6.5 and Theorem 6.2.20 of [8] show that $K$ is contractible.

Lemma 4 (Pseudo Schoenflies Lemma). A bicollared $(n-1)$ sphere $S^{n-1}$ in a pseudo-sphere $M^{n}$ is the common boundary of two psudo-cells.

MAIN ResULT. 
THEOREM 5. If $K$ is a pointlike subset of a compact manifold $M^{n}$ and $\dot{K}$ is an $(n-1)$-sphere collared on the side containing $K$, then $K$ is a pseudo-cell.

Proof. Assume $n \geqq 3$. Denote by $L$ the set $\left(\left(M^{n} \sim K\right) \cup\right.$ collar of $\dot{K}$ ). Then $L$ and $K$ are closed and their union is $M^{n}$ while their intersection is simply connected. By the Van Kampen Theorem $\pi_{1}\left(M^{n}\right)=$ $\pi_{1}(L) * \pi_{1}(K)$, where $*$ denotes the free product. Borsuk [2] has shown that every compact manifold is dominated by a polyhedron, that is there is a finite polyhedron $P$ and continuous maps $f: P \rightarrow M^{n}$ and $g: M^{n} \rightarrow P$ such that $f \circ g$ is a homotopic to $1_{M^{n}}$. It follows that $\pi_{1}\left(M^{n}\right)$ is a finitely presented group. Since $K$ is pointlike, $\pi_{1}\left(M^{n} \sim K\right)=$ $\pi_{1}(L)=\pi_{1}\left(M^{n} \sim\{p\}\right)=\pi_{1}\left(M^{n}\right)$. We have $\pi_{1}\left(M^{n}\right)=\pi_{1}(K) * \pi_{1}(L)=$ $\pi_{1}(K) * \pi_{1}\left(M^{n}\right)$. By Grusko's theorem [6], $\pi_{1}(K)$ is trivial.

To show that $\pi_{q}(K)$ is trivial for $q \leqq n$ we show that $H_{q}(K)$ is trivial for $q \leqq n-2$, then we use duality to get $H_{g}(K)=0$ for $q \leqq n$. Since $K$ and $L$ form an excisive couple we may apply the Mayer-Vietoris Sequence to get

$$
\begin{aligned}
& H_{q}(K \cap L) \rightarrow H_{q}(K) \oplus H_{q}(L) \rightarrow H_{q}(K \cup L) \rightarrow H_{q-1}(K \cap L), \\
& 1 \leqq q \leqq n-2 .
\end{aligned}
$$

Since $K \cap L$ is an $n$-annulus this sequence becomes

$$
0 \rightarrow H_{q}(K) \oplus H_{q}(L) \rightarrow H_{q}(K \cup L) \rightarrow 0,
$$

which implies that $H_{q}(K) \oplus H_{p}(L) \approx H_{q}(K \cup L)$. Since $K$ is pointlike, $H_{q}(K \cup L) \approx H_{q}(L)$. Since there is a dominating polyhedron for $M^{n}$, $H_{q}\left(M^{n}\right)$ is a finitely generated group. It follows that $H_{q}(K)$ is trivial. By the Hurewicz Isomorphism Theorem, $\pi_{q}(K)=0$ for $1 \leqq q \leqq n-2$. Let $S$ be the compact manifold obtained by sewing a cell to the boundary of $K$. Then by duality, $S$ is a homotopy sphere. By Lemma $4, K$ is contractible.

If $n=2$ then $K$ can be shown to shown to be a 2-cell by the classification theorem for compact 2-manifolds with contours for boundary.

CoRollary 6. Let $K$ be a pointlike subset of a compact manifold $M$, then $K$ lies in a pseudo-cell with sphere boundary.

Proof: Let $h: M \rightarrow M$ be the continuous map given by Lemma 2 . Let $C^{\prime}$ be a cell containing $p$ and having a bicollared boundary. Then $C^{\prime}$ is pointlike so $h^{-1}\left(C^{\prime}\right)=C$ is a pointlike subset of $M$ with bicollared sphere boundary. The previous theorem shows that $C$ is a pseudo-cell.

COROLLARY 7. In a compact manifold in which every pseudocell with sphere boundary is a cell, a pointlike subset is cellular. 
LEMmA 8. If $K$ is a pointlike subset of a compact manifold $M$, then there are infinitely many disjoint homeomorphic copies of $K$ in $M$.

Proof. Let $p \in M \sim K$ and let $h: M \sim K \rightarrow M \sim\{p\}$ be a homeomorphism. Let $h^{-1}(K)=K_{1} \subset M \sim K$. Let $g_{1}$ be a homeomorphism of $M$ onto itself such that $g_{1}(p)=p_{1} \notin K \cup K_{1}$ and $g_{1}=1$ on $K$. Let $h_{1}=g_{1} \circ h$. Then $h_{1}^{-1}\left(K_{1}\right)=K_{2}$ is homeomorphic with $k$ and

$$
h_{1}^{-1}\left(K_{1}\right) \cap\left(K_{1} \cap K\right)=\varnothing .
$$

Continuing in this fashion we get $K, K_{1}, K_{2}, \cdots$.

The complement of two disjoint pointlike subsets of a manifold $M$ need not be homeomorphic with the complement of two points in $M$; for example two linked 1-spheres in the 3-manifold of Example 1.

THEOREM 9. A pointlike subset of a compact $n$-manifold $(n \neq 4)$ is cellular.

Proof. By Corollary 6, the pointlike set lies in a pseudo-cell $P$ with sphere boundary. Sew a cell to $P$ along their boundaries to get a homotopy sphere $S^{n}$. Since the Poincare Conjecture has been proved [7] for $n \geqq 5, S^{n}$ must be a sphere. The generalized Schoenflies Theorem [3] shows that $P$ is a cell. An application of Lemma 3 completes the proof when $n \geqq 5$. If $K$ is a pointlike subset of a compact manifold $M$, then there are countably many disjoint homeomorphic copies of $K$ in $M$. Thus if $K$ is a pointlike subset of $M$ that is not cellular, then $M$ must contain countably many disjoint pseudo-cells that are not cells. If $n=3, M$ is triangulable so an application of Bing's Side Approximation Theorem [1] allows us to assume that each pseudo-cell has a polyhedral sphere boundary. Kneser [5] has shown that such a decomposition can contain only finitely many such sets that are not cells.

We note that we have a generalization of the Generalized Schoenflies theorem: If $S^{n-1}$ is a bicollared $(n-1)$-sphere that separates a compact $n$-manifold $M$ and one of the components of $M-S^{n-1}$ is pointlike, then that component is a pseudo-cell.

One should observe that the proof the Theorem 5 shows: If $K$ is a pointlike subset of an $n$-manifold $M, \pi_{m}(M)$ is finitely generated for $1 \leqq m \leqq n$, and $\dot{K}$ is an $(n-1)$-sphere collared on the side containing $K$, then $K$ is a pseudo-cell.

Using arguments like those used in the proof of Theorem 5, one can show that a compact $n$-manifold $(n \neq 4)$ can be written as the connected sum of at most finitely many nontrivial summands.

The authors wish to thank K. W. Kwun for his helpful suggestions. 
Question. If we drill countably many disjoint cells out of $S^{4}$ and sew in pseudo-cells, is the resulting space ever a manifold?

\section{BIBLIOGRAPHY}

1. R. H. Bing, Approximating surfaces from the the side, Ann. of Math. 77 (1963), 145-192.

2. K. Borsuk, Über eine Klasse von lokal zusammenhängenden Räumen, Fund. Mat. 19 (1932), 220-242.

3. M. Brown, A proof of the generalized Schoenfies theorem, Bull. Amer. Math. Soc. 66 (1960), 74-76.

4. - The monotone union of open n-cells is an open n-cell, Proc. Amer. Math. Soc. 12 (1961), 812-814.

5. H. Kneser, Gescholossen Flächen in driedimensionalen Mannigfaltigkeiten, Jahresbericht der Deutchen Mathematiker Vereinigung 38 (1929), 248-260.

6. A. G. Kurosh, The Theory of Groups, vol. II, Chelsea, New York, 1956.

7. M. H. A. Newman, The engulfing theorem for topological manifolds, Ann. of Math. 84 (1966), 555-571.

8. E. H. Spanier, Algebraic Topology, McGraw-Hill, New York, 1966.

Received March 17, 1967. This research was partially supported by the University of Idaho Research Council.

UNIVERSITY OF IDAHO 



\section{PACIFIC JOURNAL OF MATHEMATICS}

\section{EDITORS}

\author{
H. ROYDEN \\ Stanford University \\ Stanford, California
}

\author{
J. P. JANS \\ University of Washington \\ Seattle, Washington 98105
}

J. DugundJI

Department of Mathematics

Rice University

Houston, Texas 77001

RICHARD ARENS

University of California

Los Angeles, California 90024

\section{ASSOCIATE EDITORS}
E. F. BECKENBACH
B. H. NeumanN
F. WOLF
K. YOSIDA

\section{SUPPORTING INSTITUTIONS}

\author{
UNIVERSITY OF BRITISH COLUMBIA \\ CALIFORNIA INSTITUTE OF TECHNOLOGY \\ UNIVERSITY OF CALIFORNIA \\ MONTANA STATE UNIVERSITY \\ UNIVERSITY OF NEVADA \\ NEW MEXICO STATE UNIVERSITY \\ OREGON STATE UNIVERSITY \\ UNIVERSITY OF OREGON \\ OSAKA UNIVERSITY \\ UNIVERSITY OF SOUTHERN CALIFORNIA
}

\author{
STANFORD UNIVERSITY \\ UNIVERSITY OF TOKYO \\ UNIVERSITY OF UTAH \\ WASHINGTON STATE UNIVERSITY \\ UNIVERSITY OF WASHINGTON \\ AMERICAN MATHEMATICAL SOCIETY \\ CHEVRON RESEARCH CORPORATION \\ TRW SYSTEMS \\ NAVAL ORDNANCE TEST STATION
}

\footnotetext{
Mathematical papers intended for publication in the Pacific Journal of Mathematics should be in typed form or offset-reproduced, double spaced with large margins. Underline Greek letters in red, German in green, and script in blue. The first paragraph or two must be capable of being used separately as a synopsis of the entire paper. It should not contain references to the bibliography. Manuscripts may be sent to any one of the four editors. All other communications to the editors should be addressed to the managing editor, Richard Arens, University of California, Los Angeles, California 90024.

Each author of each article receives 50 reprints free of charge; additional copies may be obtained at cost in multiples of 50 .

The Pacific Journal of Mathematics is published monthly. Effective with Volume 16 the price per volume (3 numbers) is $\$ 8.00$; single issues, $\$ 3.00$. Special price for current issues to individual faculty members of supporting institutions and to individual members of the American Mathematical Society: $\$ 4.00$ per volume; single issues $\$ 1.50$. Back numbers are available.

Subscriptions, orders for back numbers, and changes of address should be sent to Pacific Journal of Mathematics, 103 Highland Boulevard, Berkeley 8, California.

Printed at Kokusai Bunken Insatsusha (International Academic Printing Co., Ltd.), 7-17, Fujimi 2-chome, Chiyoda-ku, Tokyo, Japan.

PUBLISHED BY PACIFIC JOURNAL OF MATHEMATICS, A NON-PROFIT CORPORATION

The Supporting Institutions listed above contribute to the cost of publication of this Journal, but they are not owners of publishers and have no responsibility for its content or policies.
} 


\section{Pacific Journal of Mathematics}

\section{Vol. 24, No. $3 \quad$ July, 1968}

Duane W. Bailey, On symmetry in certain group algebras ............ 413

Lawrence Peter Belluce and Surender Kumar Jain, Prime rings with a one-sided ideal satisfying a polynomial identity ................ 421

L. Carlitz, A note on certain biorthogonal polynomials ............. 425

Charles O. Christenson and Richard Paul Osborne, Pointlike subsets of a manifold ......................................... 431

Russell James Egbert, Products and quotients of probabilistic metric

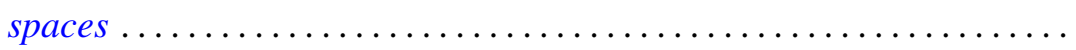

Moses Glasner, Richard Emanuel Katz and Mitsuru Nakai, Bisection into small annuli ..................................... 457

Karl Edwin Gustafson, A note on left multiplication of semigroup

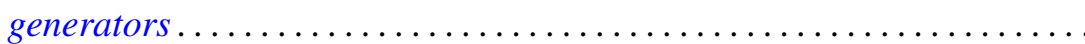

I. Martin (Irving) Isaacs and Donald Steven Passman, A characterization of groups in terms of the degrees of their characters. II ............. 467

Howard Wilson Lambert and Richard Benjamin Sher, Point-like

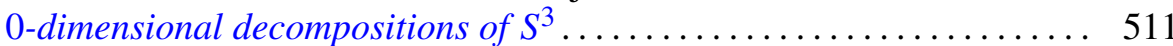

Oscar Tivis Nelson, Subdirect decompositions of lattices of width two ..... 519

Ralph Tyrrell Rockafellar, Integrals which are convex functionals . . . . . . . 525

James McLean Sloss, Reflection laws of systems of second order elliptic differential equations in two independent variables with constant coefficients ...

Bui An Ton, Nonlinear elliptic convolution equations of Wiener-Hopf type in a bounded region

Daniel Eliot Wulbert, Some complemented function spaces in $C(X)$

Zvi Ziegler, On the characterization of measures of the cone dual to a generalized convexity cone. 